\title{
Article \\ Multichannel Real-Time Electronics Platform for the Estimation of the Error in Impact Localization with Different Piezoelectric Sensor Densities ${ }^{\dagger}$
}

\author{
Lorenzo Capineri *(D), Andrea Bulletti (1) and Eugenio Marino Merlo
}

check for updates

Citation: Capineri, L.; Bulletti, A.; Marino Merlo, E. Multichannel Real-Time Electronics Platform for the Estimation of the Error in Impact Localization with Different Piezoelectric Sensor Densities . Appl. Sci. 2021, 11, 4027. https://doi.org /10.3390/app11094027

Academic Editor: Habil.

Michel Darmon

Received: 26 March 2021

Accepted: 27 April 2021

Published: 28 April 2021

Publisher's Note: MDPI stays neutral with regard to jurisdictional claims in published maps and institutional affiliations.

Copyright: (c) 2021 by the authors. Licensee MDPI, Basel, Switzerland. This article is an open access article distributed under the terms and conditions of the Creative Commons Attribution (CC BY) license (https:/ / creativecommons.org/licenses/by/ $4.0 /)$.
Department of Information Engineering, University of Florence, 50139 Florence, Italy; andrea.bulletti@unifi.it (A.B.); eugenio.marinomerlo@unifi.it (E.M.M.)

* Correspondence: lorenzo.capineri@unifi.it

+ This paper is an extended version of our paper published in A. Bulletti, E. M. Merlo and L. Capineri, "Analysis of the accuracy in impact localization using piezoelectric sensors for Structural Health Monitoring with multichannel real-time electronics," 2020 IEEE 7th International Workshop on Metrology for AeroSpace (MetroAeroSpace), Pisa, Italy, 22-24 June 2020.

\begin{abstract}
The work presents a structural health monitoring (SHM) electronic system with real-time acquisition and processing for the determination of impact location in laminate. The novelty of this work is the quantitative evaluation of impact location errors using the Lamb wave guided mode $\mathrm{S}_{0}$, captured and processed in real-time by up to eight piezoelectric sensors. The differential time of arrival is used to minimize an error function for the position estimation. The impact energy is correlated to the amplitudes of the antisymmetric $\left(\mathrm{A}_{0}\right)$ mode and the electronic design is described to avoid saturation for signal acquisition. The same electronic system is designed to acquire symmetric $\left(\mathrm{S}_{0}\right)$ low level signals by adequate gain, bandwidth, and signal-to-noise ratio. Such signals propagate into a $1.4 \mathrm{~mm}$ thick aluminum laminate at the group velocity of $5150 \mathrm{~m} / \mathrm{s}$ with frequency components above $270 \mathrm{kHz}$, and can be discriminated from the $\mathrm{A}_{0}$ mode to calculate accurately the differential arrival time. The results show that the localization error stabilizes at a value comparable with the wavelength of the $S_{0}$ mode by increasing the number of sensors up to six, and then remains constant at up to eight sensors. This suggests that a compromise can be found between sensor density and localization error.
\end{abstract}

Keywords: real-time electronics; structural health monitoring; Lamb wave; piezoelectric sensors; impact localization; ultrasonic guided waves

\section{Introduction}

Structural health monitoring (SHM) has been the subject of different studies in the non-destructive testing (NDT) field aiming to identify the location of an impact point using piezoelectric sensors. Many plate-like geometries made in fiber-reinforced composite (CFRP) or aluminum — commonly used in aerospace structures-are suitable to support the guided propagation of ultrasound-guided waves over long distances; for plate-like structures a few mm thick we refer to extensional and flexural modes of Lamb waves. The possibility of identifying damage on a structure by piezoelectric sensor systems allows for the determination of its integrity, thus reducing downtime and maintenance costs. SHM is important because, by continuous monitoring, it permits real-time detection of damage in a structure, particularly of safety-critical components typical of the application of such compounds in aircrafts [1-5]. A real-time SHM system with a network of passive sensors was proposed to detect low- and high-impact events. For each event, the conversion of kinetic energy into propagating ultrasonic guided waves occurs and acoustic emission (AE) localization methods can be applied using piezoelectric transducers. Once a localization is performed, the SHM system can be switched to the active mode operation to investigate a 
restricted area around the estimated impact position. An array of sensors and real-time multichannel electronic processing are the enabling technologies for the application of SHM in aerospace, energy conversion, transportation, and automotive industries.

In the literature, different strategies for locating impact positions using Lamb waves were investigated with reference to the extraction of the differential time of arrival (DToA). Tobias in [6] used a triangulation technique; Ciampa and Meo in [7] evaluated the DToA with an algorithm based on continuous wavelet transform (CWT); Shukri Mohd et al. in [8] used a method based on wavelet transform analysis and modal location (WTML) with four sensors; Shenxin Yin et al. in [9] used eight sensors bonded in a Z-shaped arrangement. While CWT allows more sophisticated signal processing for the separation of the flexural and extensional dispersive modes, in a multisensory system with several nodes simpler solutions-like the first arrival signal detection with threshold method or short-time Fourier transform (STFT) - must also be considered. The latter requires less computational resources than CWT but does not permit the trade-off between the arrival time and spectral content.

For complex three-dimensional structures, analysis of the DToA, relative to propagation along multiple paths, is not straightforward and is thus difficult to determine with analytical models. In these cases, multiple sensors and artificial intelligence (AI) are often used; for example, the system based on neural networks presented by Worden et al. [10] used up to 17 sensors. Carrino et al. [11,12] proposed an innovative method based on nonlinear Lamb waves for locating disbonds in single-lap joints that uses PZT signals processed with a baseline-free algorithm and the localization of artificial defects placed inside the area, delimited by four PZT sensors.

Other strategies for locating impact positions, without knowledge of the characteristics of the material under test, are also reported in [13,14]. In a previous work, Kundu et al. [14] presented a study that considered different shapes of the wave front generated during an acoustic event and developed a methodology to localize the acoustic source in an anisotropic plate from those wave front shapes: an elliptical wave front shape-based technique was developed first, followed by the development of a parametric curve-based technique for non-elliptical wave front shapes. This methodology does not assume a straight-line wave propagation path and can predict the source location without any knowledge of the elastic properties of the material; however, this study reported only a theoretical approach without the development of a real-time system to validate the proposed technique.

In [15], the authors of this work presented a guided-wave ultrasound SHM system based on linear arrays of interdigital piezopolymer transducers bonded to a composite pressure vessel for spacecrafts. In that system, interdigital transducers were adopted to perform both damage assessment and impact detection/localization using a simple fixed-threshold technique. In a related research development [16], the authors presented a novel approach to estimate the DToA between the impact response signals collected by a triplet of sensors, overcoming the limitations of classical methods that rely on amplitude thresholds calibrated for a specific sensor type. Finally, in [17] is proposed a simple laboratory procedure based on a set-up with a pair of sensors that are symmetrically placed with respect to the impact point to estimate the uncertainty of the DToA and the propagation velocity.

In this work we have upgraded the real-time electronic system reported in [15] that allows for the accommodation of analog front-end electronic characteristics thanks to its modular design; different sensor types (piezoceramic, piezocomposites, and piezopolymers) need custom analog front-ends (AFE) to match their electrical impedance, sensitivity, and bandwidth. In this work, we designed and implemented 16 new receiver boards to collect information of the propagated extensional $S_{0}$ mode Lamb waves thanks to the improved bandwidth, adjustable gain, and signal-to-noise ratio. This new feature allowed us to overcome the limitation of using information for the localization carried by the higher level, but slower, $\mathrm{A}_{0}$ mode. The detection of small amplitude $(\mathrm{mV}-10 \mathrm{mV}) \mathrm{S}_{0}$ mode signal 
with threshold method simplifies the electronic design with benefits also to power consumption and rapid event detection. As observed in $[18,19], \mathrm{A}_{0}$ mode propagates on longer paths with low attenuation but at lower propagation velocity and exhibits dispersion phenomena in the low-frequency range. Such characteristics complicate the interpretation of the impact-generated signals, especially considering large structures and multiple reflections from boundary, where in many cases $\mathrm{S}_{0}$ is preferred. Moreover, the importance of sensor networks for aircraft SHM was reported in [20], considering the large dimensions and the trade-off between system performance, reliability, and cost.

The study presented in this paper differs from previously cited works because the aim was not to present the best method or technique for locating impact positions, but to report the analysis of errors on impact localization (see Section 2) using real-time electronics; this paper also evaluates the influence of the number of passive PZT sensors used (see Section 3) on the position error. The real-time electronics can also acquire multiple impacts on the same point for statistical analysis. We present the results of experimental tests with low-energy impact on a $1.4 \mathrm{~mm}$ thick aluminum plate using Physik Instrumente P-876.SP1 piezoceramic transducers, varying the number of channels from four to eight. The signals were then used to find the minimum of a cost function for the localization of the impact and, therefore, the evaluation of the positioning error relative to the size of the investigation area and the number of passive channels used. Finally, to help the reader in finding selected topics we include, a list of acronyms used in this paper.

\section{Impact Detection and Localization}

Considering a plate-like structure, a point impact on the surface will determine a mechanical response in the form of elastic waves of various natures propagating outward from the point of contact. Our work focused on detecting low-energy impacts (from 35 to $600 \mathrm{~mJ}$ ) that did not result in permanent damage to the aluminum plate. Low-energy impacts are of interest for laboratory testing of system prototypes because they are nondestructive, while larger energy ( $>1 \mathrm{~J}$ ) impacts are used for real-life SHM applications. The implication of sensor choice, regarding sensitivity and impact energy, is discussed in the next section.

In general, it is possible to detect the location of an impact event by measuring the DToAs of the elastic response wave front to a set of three or more sensors with knowledge of their position $\left(\mathrm{x}_{\mathrm{i}}, \mathrm{y}_{\mathrm{i}}\right)$ on a planar structure. Using these data, the impact location can be numerically triangulated. Our system estimated the DToA of impact-generated elastic waves using a simple amplitude threshold thanks to the remarkable (better than $70 \mathrm{~dB}$ ) signal-to-noise ratio (SNR) of the analog front-end.

The developed system performed impact detection by running in passive (listening) mode, i.e., by continuously sampling the transducer signals in a circular buffer, while waiting for a triggering event (a threshold-crossing) to occur. This threshold level (six times the root-mean-square noise level) had to be verified during the experimental tests to avoid spurious triggering, while maintaining a good sensitivity to low-energy impacts. As the data acquisition hardware detected an impact threshold-crossing event, it immediately froze the circular buffer and transferred the data to the acquisition system.

We defined a region of interest (ROI) of the aluminum plate equal to $250 \mathrm{~mm} \times 240 \mathrm{~mm}$ (see Figure 1). This area was divided into a uniform grid of points spaced $1 \mathrm{~mm}$ apart with coordinates $\left(x_{p}, y_{p}\right)$ for the subsequent analysis. For each of those points, the impact localization algorithm processed the received signals to extract the differential DToAs and calculated the value of the error function $E\left(x_{p}, y_{p}\right)$ [15-17]. Once the error function (1) was calculated for the whole grid, the impact point was assigned by the absolute minimum error value criterion. The error function $\mathrm{E}\left(\mathrm{x}_{\mathrm{p}}, \mathrm{y}_{\mathrm{p}}\right)$ presents a minimum and has a monotonic behavior. In such cases, one can extrapolate a best-guess position of the impact by calculating the centroid of the points having a value within $1 \%$ of the absolute minimum. 
The choice to use a grid of points spaced $1 \mathrm{~mm}$ apart was a good compromise between the accuracy of estimation of the impact point and the necessity to elaborate signals in real-time and immediately show the results on a PC display.

$$
E\left(x_{p}, y_{p}\right)=\sum_{i=1}^{N_{T}-1} \sum_{j=i+1}^{N_{T}}\left|\frac{\left(t_{i}-t_{j}\right) v-\left(\sqrt{\left(\mathrm{x}_{\mathrm{i}}-\mathrm{x}_{\mathrm{p}}\right)^{2}+\left(\mathrm{y}_{\mathrm{i}}-\mathrm{y}_{\mathrm{p}}\right)^{2}}-\sqrt{\left(\mathrm{x}_{\mathrm{j}}-\mathrm{x}_{\mathrm{p}}\right)^{2}+\left(\mathrm{y}_{\mathrm{j}}-\mathrm{y}_{\mathrm{p}}\right)^{2}}\right)}{\sqrt{\left(\mathrm{x}_{\mathrm{i}}-\mathrm{x}_{\mathrm{j}}\right)^{2}+\left(\mathrm{y}_{\mathrm{i}}-\mathrm{y}_{\mathrm{j}}\right)^{2}}}\right|
$$

Here $N_{T}$ is the total number of installed sensors whose positions are defined by the coordinates $P_{i}=\left(x_{i}, y_{i}\right)$ and $P_{j}=\left(x_{j}, y_{j}\right)$. The corresponding measured time of flights for each sensor are $t_{i}$ and $t_{j}$, respectively.

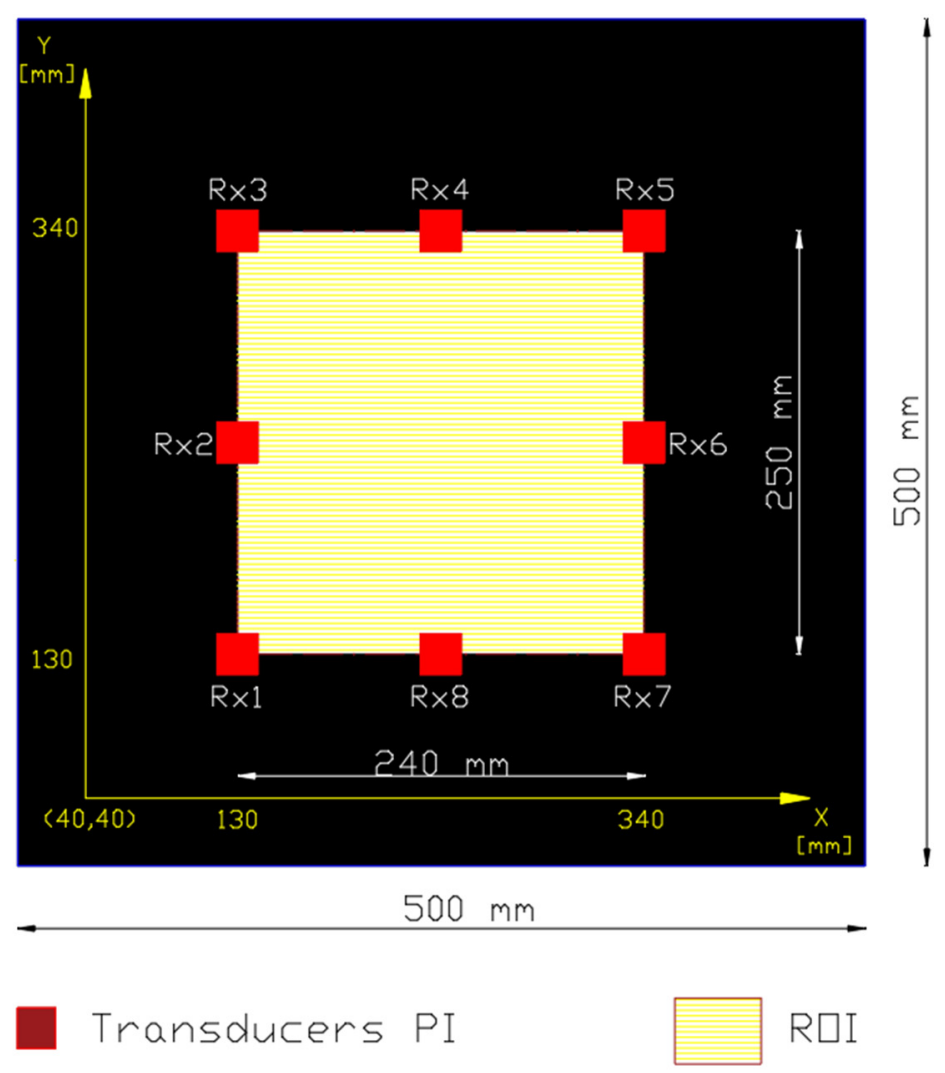

Figure 1. The $1.4 \mathrm{~mm}$ thick aluminum plate with 8 -sensor arrangement (red squares). The picture also indicates the ROI (yellow area) and the area outside the ROI (black area) that corresponds to the aluminum laminate's dimensions.

\section{Experimental Set-Up and Electronic Equipment}

This section describes the electronic system designed for SHM application using multiple sensors in passive mode. The system can also be switched automatically to active mode for damage assessment using interdigital transducers as reported in [15].

\subsection{Electronic Instrument Design for SHM Evaluation}

The main architecture and design of the electronic instrument developed as part of this work has been presented in [21]. The instrument consists of a 16-channel device fully programmable in active or passive modes of operation. One of the main characteristics of this electronic system is the real-time acquisition and processing of ultrasonic signals received by 16 channels, which is an advantage in research of sensor layouts with more 
than 3 or 4 channels; the latter being quite a common experimental situation made available with use of a digital oscilloscope.

For the passive operation mode treated in this work, we designed 16 new receiver boards that easily replaced the previous ones thanks to the modular architecture of the system. A block-scheme of the new board is shown in Figure 2. The input attenuator is a fundamental block to adjust for different sensor sensitivities and impact energies. For example, impacts with 10 to $100 \mathrm{~mJ}$ of energy can be monitored with the $6.5 \mathrm{~mm}$ PVDF circular type (see $[16,17]$ ), producing voltage signals in the range of $100 \mathrm{mV}$ to $1 \mathrm{~V}$, while the highest sensitivity of piezoceramic sensors, such as the Physik Instrumente P-876.SP1, allows monitoring of signals up to $300 \mathrm{mVpp}$ from the low-energy impact hammer or free-falling sphere typically used during non-destructive laboratory tests. The piezoceramic material of these types of sensors has a high electromechanical coupling factor and high sensitivity.
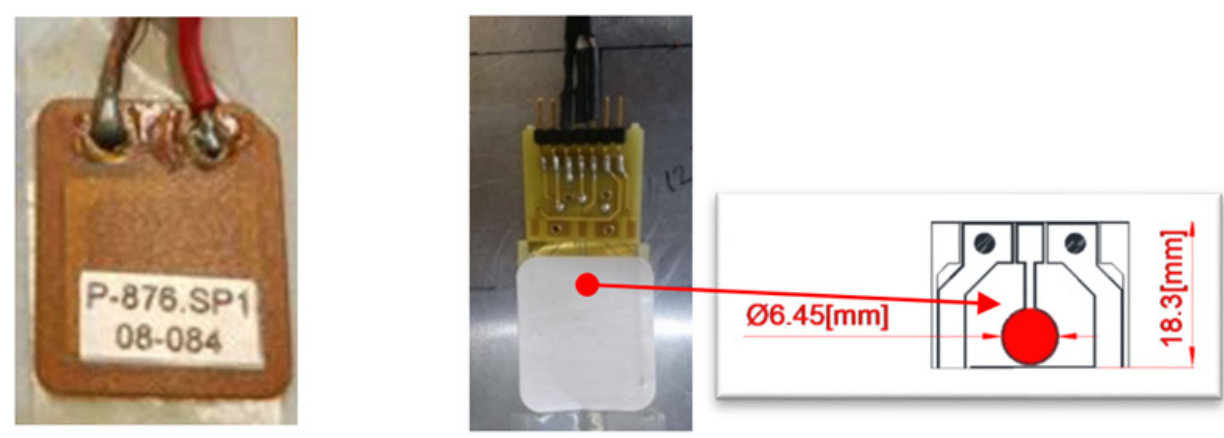

Figure 2. (Left) Piezoceramic sensor PI_876.SP1. Piezopolymer (PVDF) sensor protected by a white adhesive label and electrical connections on a PCB. (Right) Circular element of $6.45 \mathrm{~mm}$ diameter shown by the red circle on the CAD drawing.

The two different types of piezoelectric sensors used are shown in Figure 2, and their signal output amplitude was measured by direct connection to a digital oscilloscope; the comparison of the amplitudes is reported in Figure 3 for different impact energies from 0 to $45 \mathrm{~mJ}$. In Figure 3, we can observe different energy values, $\mathrm{U}=\mathrm{m} \times \mathrm{g} \times \mathrm{h}$, corresponding to the same value of mass, $m$, and acceleration due to gravity, $g$, of the impacting steel sphere: these different values were obtained according to the above relationship by varying the falling height, $\mathrm{h}$.

These amplitudes are relative to the peak amplitude of the large $A_{0}$ mode which is generated by the impact and has low-frequency components, typically below $50 \mathrm{kHz}$ as predicted by Ross in [22]. The data reported in Figure 3 are important in making a decision as to the programmable threshold value depending on the expected impact energy. In this way, the real-time electronics capture the signals with a predefined minimum energy and, thanks to the programmed pre-trigger time, the signal trace also contains the information of signals preceding the large $\mathrm{A}_{0}$ mode. This is a relevant feature of the electronic system because higher frequency components of the $A_{0}$ and $S_{0}$ propagate at higher velocities, as explained in Section 4.

The electronic analog front-end (AFE) (see Figure 4) is mainly composed of a programmable input attenuator (from 0 to $-20 \mathrm{~dB}$ ), a unity gain buffer based on an LF347 op amp, a non-inverting amplifier with voltage gain of $25 \mathrm{~dB}$ based on an LM6172 op amp, and a band pass filter (BPF). The BPF is designed to obtain an active (op amp LM6172) second-order Bessel HPF with the cut-off frequency (fc1) equal to $270 \mathrm{kHz}$ at $-3 \mathrm{~dB}$, and a passive first-order LPF with the cut-off frequency (fc2) equal to $800 \mathrm{kHz}$ at $-3 \mathrm{~dB}$. The last filtering block was inserted to limit the in-band noise; the noise measurements of the output of the analog chain with a standard 50-ohm input impedance for two different prototype boards are reported in Table 1. 


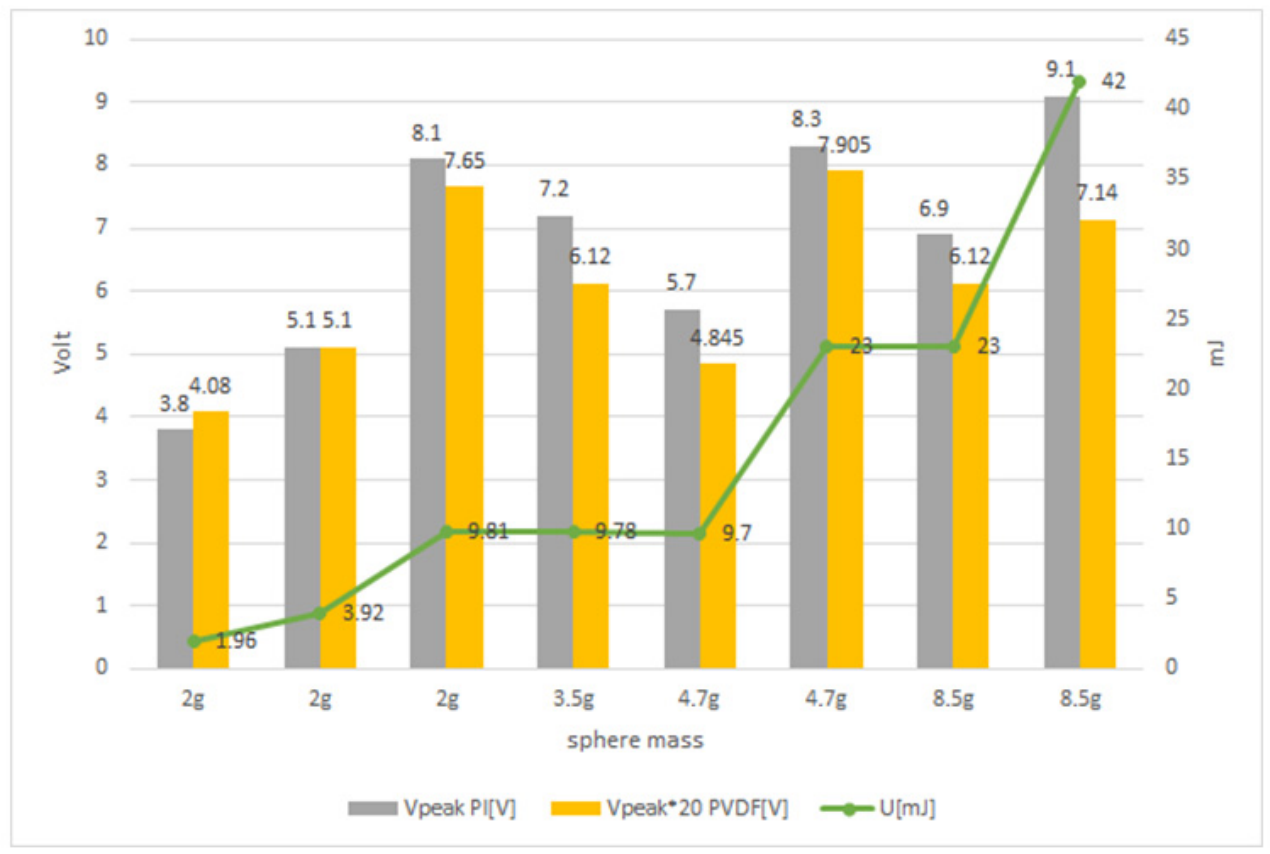

Figure 3. Peak voltage from impacts on the aluminum laminate generated by steel balls with mass ranging from 2 to $8.5 \mathrm{~g}$ in free-fall. The sensitivity of the two different sensors (see Figure 2) can be compared by the output voltages: the PVDF sensor amplitudes were multiplied by a constant factor, 20, to allow for comparison with the trend of the PZT amplitude. We can observe the good correlation between impact energy and output voltage.

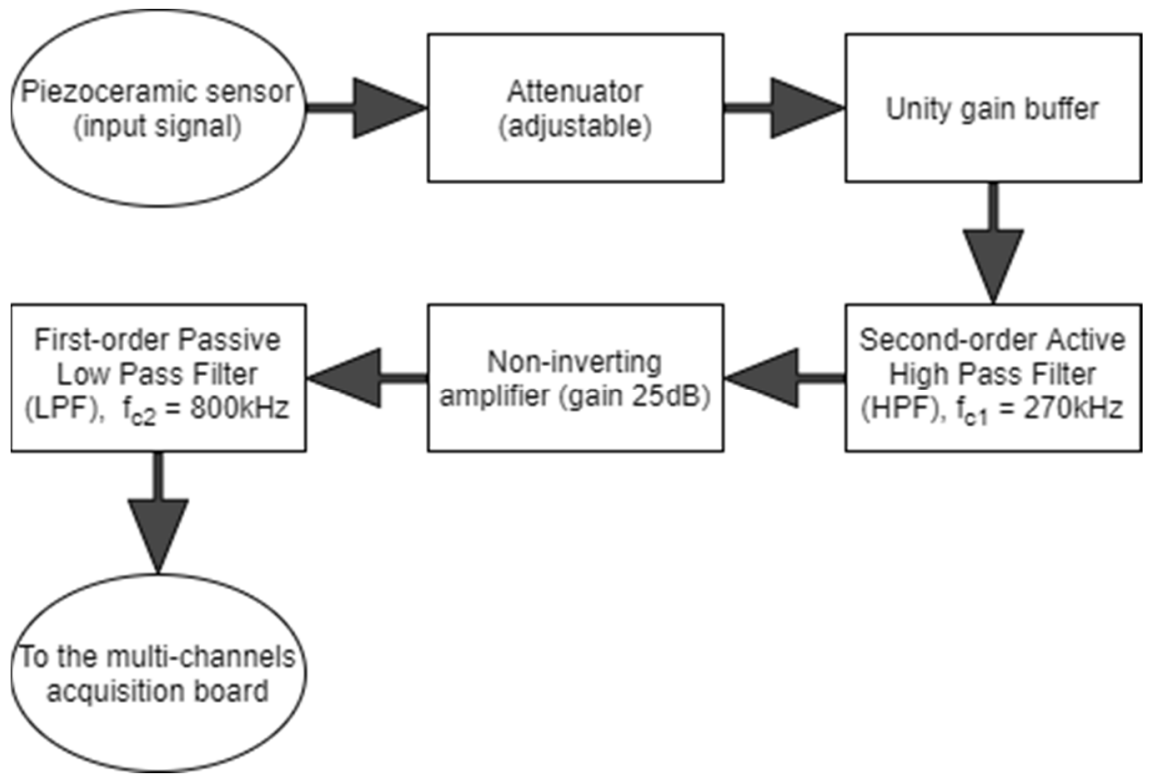

Figure 4. The block-scheme of the new design of the receiver board.

Table 1. $V_{r m s}$ output noise of the front-end channel with input impedance $50 \Omega$ and attenuator selected at $0 \mathrm{~dB}$.

\begin{tabular}{ccc}
\hline & $V_{\text {rms }}$ out Front-End \#1 & $V_{\text {rms }}$ out Front-End \#2 \\
\hline Zin $=\mathbf{5 0} \Omega$ & $1 \mathrm{~m} \mathrm{~V}$ & $1 \mathrm{~m} \mathrm{~V}$ \\
\hline
\end{tabular}


These values are adequate considering the low-voltage analog-to-digital converter (ADC) has 12-bit resolution and an input dynamic of $3.3 \mathrm{~V}$ present on the electronic platform. Moreover, we observed that the programmable attenuator is necessary to limit the input signals in the AFE chain to avoid saturation and to protect the first amplifier from large signals exceeding the allowable common mode range at the operating frequency.

The main difference, with respect to the previous version [21], is the versatility of the receiver, which can be programmed to receive ultrasonic impact signals that in general include $\mathrm{S}_{0}$ and $\mathrm{A}_{0}$ modes. This makes it possible to receive higher frequencies, up to $270 \mathrm{kHz}$, with high signal-to-noise ratio $(10 \mathrm{~dB})$, to analyze $\mathrm{S}_{0}$ Lamb modes that propagate at higher velocities, with respect to $\mathrm{A}_{0}$ modes, and with lower amplitude.

An example of the output signal after the analog processing stage is shown in Figure 5. The fast $\mathrm{S}_{0}$ mode and the high-frequency component of the $\mathrm{A}_{0}$ mode are now present in the signal. The early $\mathrm{S}_{0}$ mode signal is used later for the calculation of DToAs for the impact localization by triangulation algorithm.

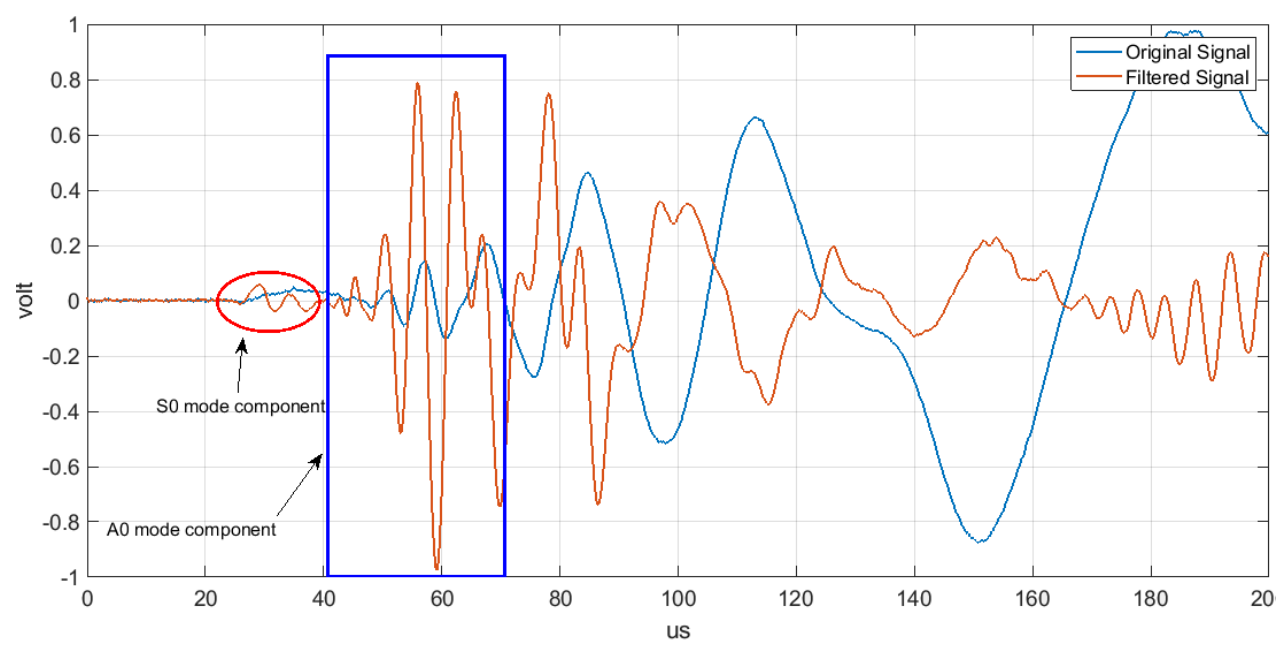

Figure 5. Comparison between original signal and filtered signal with high-pass second-order filter, with cut-off frequency $270 \mathrm{kHz}$ followed by a $25-\mathrm{dB}$ gain amplifier (see Figure 4). The signals were normalized and the distance between the impact point and the sensor was $10 \mathrm{~cm}$. The two ultrasonicguided modes $\mathrm{A}_{0}$ (marked by blue box) and $\mathrm{S}_{0}$ (marked by red oval) are well discriminated with high SNR.

\subsection{Experimental Set-Up}

In Figure 6 (right), the main blocks and connection of the SHM system are described. The main characteristics of the data acquisition system are listed below. Programmable parameters:

- VGA gain from 54 to $90 \mathrm{~dB}$ to adjust for different sensor sensitivities

- High voltage, up to $100 \mathrm{~V}$, for active mode operation

- Square wave burst (1-16 cycles), up to $500 \mathrm{kHz}$, for active mode operation Data acquisition system characteristics:

- 12-bit resolution

- 16 channels at $20 \mathrm{MSps}$

- $\quad$ Storage: 1 MS per channel

The main components of the block diagram for the data acquisition system are the:

(1) sensor layout installed on the plate-like structure, coordinate reference system, and

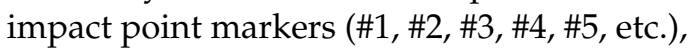

(2) signal-conditioning electronics,

(3) multichannel VGA with ADC evaluation module (Texas Instruments AFE5851EVM, Dallas, TX, USA), and 


\section{(4) Spartan-6 FPGA evaluation card (Xilinx SP605).}

The experimental set-up adopted is reported in Figure 6 (left), where only 8 out of 16 channels were programmed for passive monitoring of impacts. Figure 6 (left) shows the low-energy hammer impactor in the center of the aluminum plate where the eight piezoelectric sensor arrangement was placed. The eight sensors were placed along the perimeter of the ROI (see Figure 1) and their arrangement is shown in the experimental results section below.

The high number of sensors were also able to reveal impacts outside the ROI, up to the full size of the aluminum plate. The four edges of the aluminum plate were covered by colored play dough (see the colored edges in Figure 6) to avoid spurious reflections of the ultrasonic signal due to the edges. This simple method turned out to be necessary to manage boundary reflection due to the free edges of the plate for estimation of impact position carried out outside of the ROI. The problem concerning multiple reflections from the plate edges can also be addressed by selecting a time according to the geometry of the plate-like structure [17]. More clever algorithms exploit, also, the information retrieved by multiple reflections from boundaries to minimize the number of sensors installed on the structure [23].
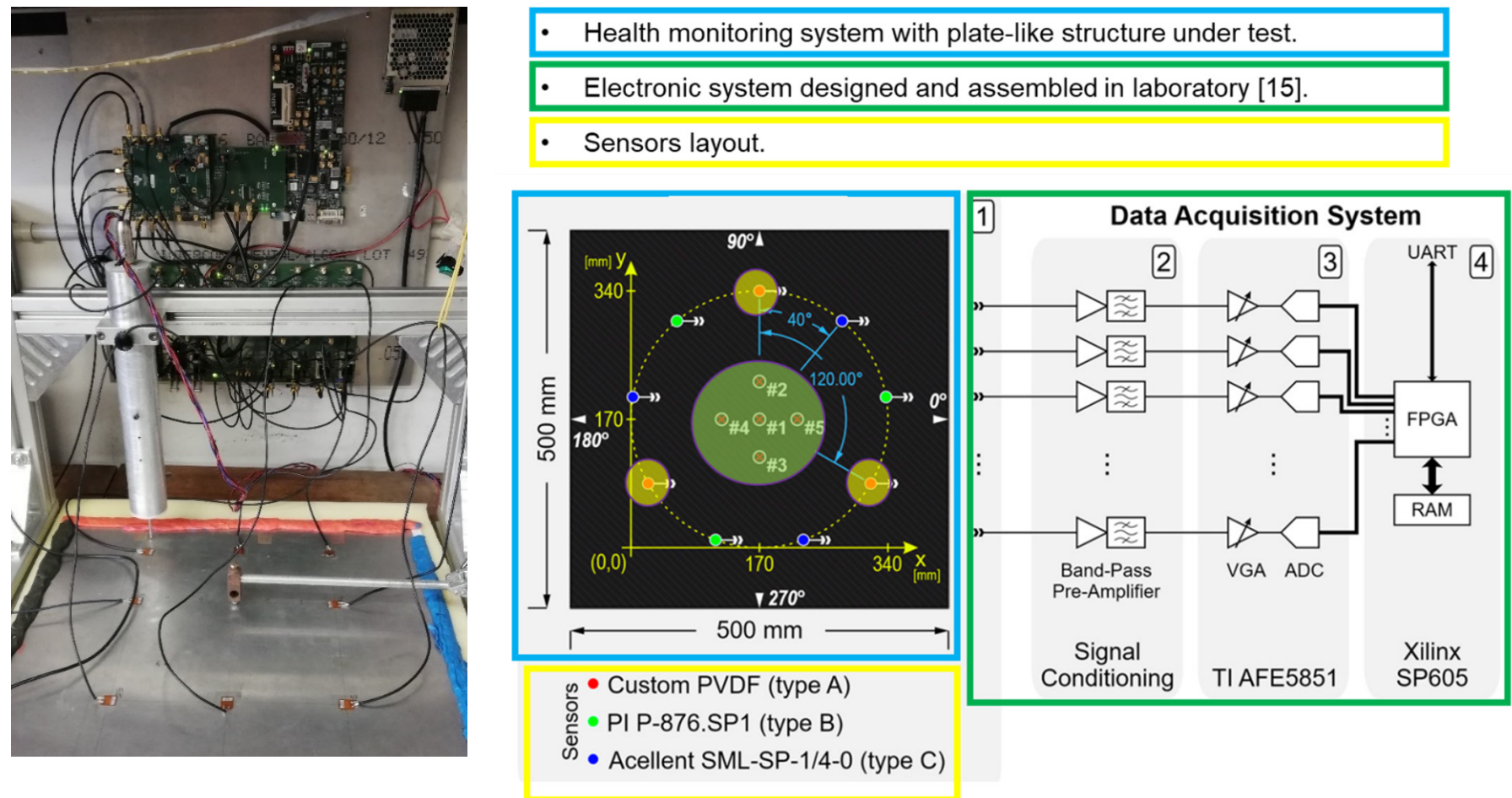

Figure 6. Experimental set-up with the eight piezoceramic transducers attached with a bi-adhesive tape to a $1.4 \mathrm{~mm}$ aluminum plate, shown in the bottom of the figure, and the real-time electronic acquisition system, shown in the top. In the center of the plate, the low-energy hammer impactor is visible. The figure also shows the colored play dough covering the edges of the aluminum plate; this method was adopted to attenuate the signals received from multipath. Reproduced from [24].

The next section reports the analysis of different errors obtained for different impact points (inside and outside the ROI) and for different numbers of channels used to find the minimum of function (1).

\section{Experimental Results}

The new custom real-time acquisition system was tested in a series of low-energy impact loading experiments so as to not damage the laminate. The aim of those experiments 
was to verify the actual capabilities of the SHM system (i.e., the localization accuracy) processing signals acquired from different numbers of sensors.

The fundamental symmetric $\left(\mathrm{S}_{0}\right)$ and anti-symmetric $\left(\mathrm{A}_{0}\right)$ group velocity dispersion curves for an aluminum plate with thickness of $1.4 \mathrm{~mm}$ are shown in Figure 7 . These curves were obtained with the LAMB MATLAB toolbox GMM calculator. From Figure 7 we can evaluate the propagation velocity of the $S_{0}$ mode. This value was used to solve Equation (1). For our experiments we considered the propagation velocity $\mathrm{v}=5150 \mathrm{~m} / \mathrm{s}$, corresponding to a frequency of $\mathrm{fS} 0=650 \mathrm{kHz}$, which falls into a frequency range where the dispersive behavior of $S_{0}$ mode is negligible but still retains a small wavelength $\mathrm{v} / \mathrm{fS} 0=8 \mathrm{~mm}$.

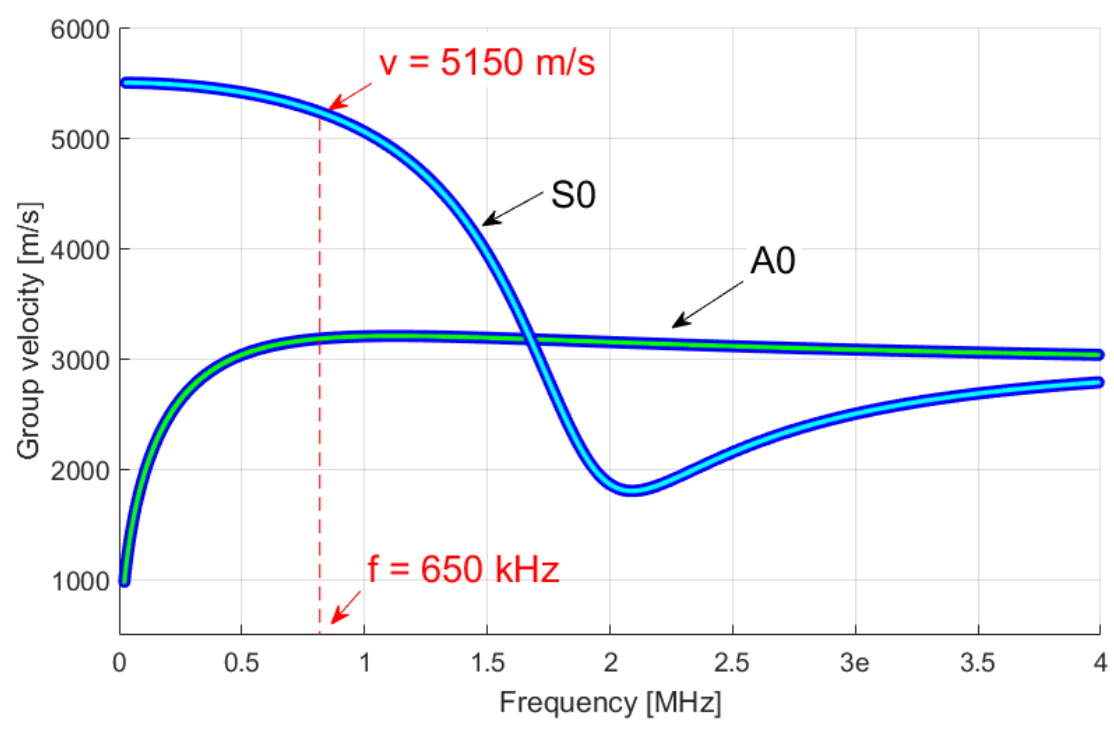

Figure 7. Viewgraph showing the calculated $S_{0}$ and $A_{0}$ group velocity dispersion curves for an aluminum plate with thickness of $1.4 \mathrm{~mm}$.

We performed three impacts: impact P1 with position $x_{i}=190 \mathrm{~mm}, y_{i}=150 \mathrm{~mm}$, performed inside the ROI, delimited by sensors; impact P2 with position $x_{i}=193 \mathrm{~mm}$, $y_{i}=32 \mathrm{~mm}$, performed outside the ROI; and impact P3 with position $x_{i}=200 \mathrm{~mm}$, $\mathrm{y}_{\mathrm{i}}=40 \mathrm{~mm}$, performed outside the ROI.

In Figures 8-13 we report the results for impacts inside and outside the ROI.

In detail, Figure 8 shows the performance of the acquisition system detecting impact point P1 and processing the signal acquired by only four sensors: $R \times 1, R \times 3, R \times 5$, and $R \times 7$, whereas Figure 9 shows the result obtained by detecting impact point $\mathrm{P} 1$ and processing the signal acquired by all eight sensors (Rx1-Rx8). The estimated position of the impact point obtained by processing signals acquired by only four sensors ( $\mathrm{P} 1^{\prime}-1$ point) is also reported in Figure 8, whereas Figure 6 shows the estimated position of the impact point obtained by processing signals acquired by all sensors ( $\mathrm{P1}^{\prime}-2$ point). To evaluate the trade-off between sensor density of an SHM system and error in impact localization, we processed the signal acquired by six sensors $R \times 1, R \times 2, R \times 3, R \times 5, R \times 6$, and $R \times 7$, and the estimated position of the impact point (P1'-3 point) is reported in Figure 10. 


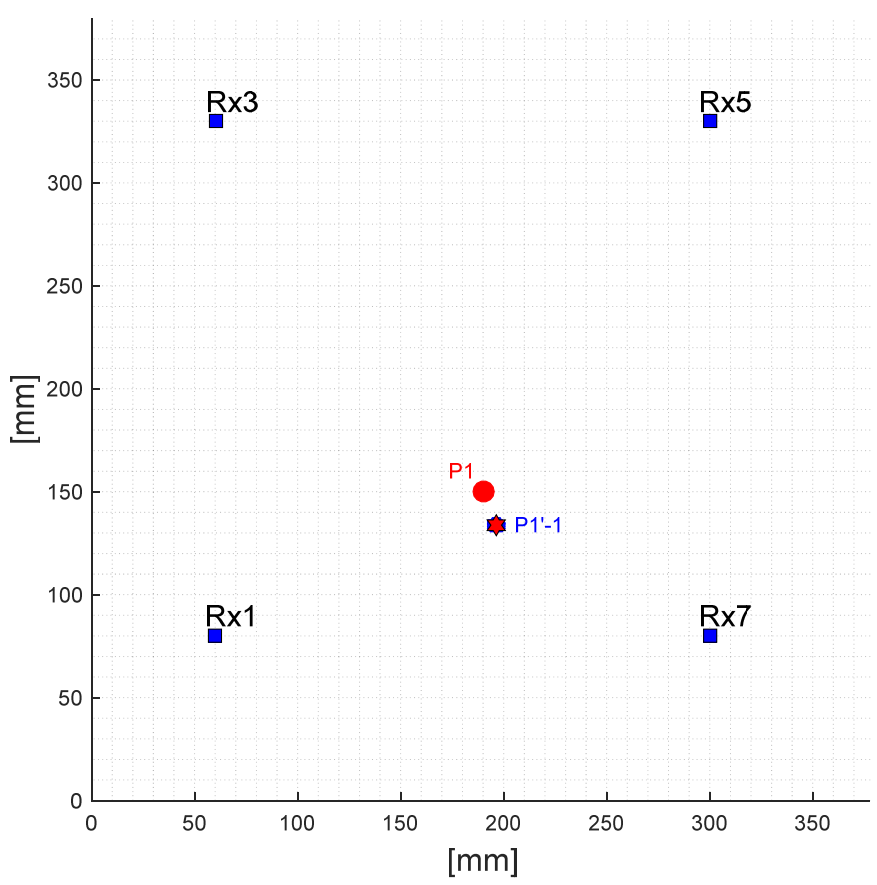

Figure 8. Position of impact P1 (red circle) inside the ROI with coordinates $x_{i}=190 \mathrm{~mm}, y_{i}=150 \mathrm{~mm}$. The detected impact position P1'-1 (red star) with coordinates $x_{p}=196 \mathrm{~mm}, y_{p}=134 \mathrm{~mm}$ was obtained by processing the ultrasonic signals acquired by only four sensors ( $R \times 1, R \times 3$, $R \times 5$, and $R \times 7$ ). Reproduced from [24].

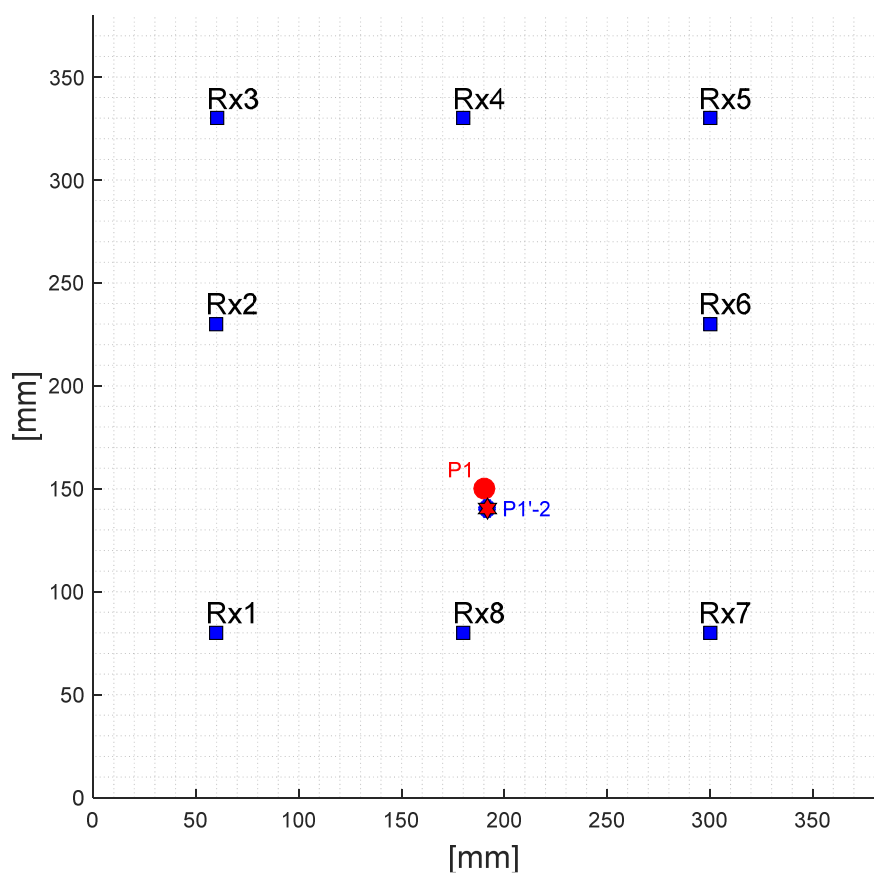

Figure 9. Position of impact P1 (red circle) inside the ROI with coordinates $x_{i}=190 \mathrm{~mm}, y_{i}=150 \mathrm{~mm}$. The detected impact position $\mathrm{P} 1^{\prime}-2$ (red star) with coordinates $\mathrm{x}_{\mathrm{p}}=192 \mathrm{~mm}, \mathrm{y}_{\mathrm{p}}=141 \mathrm{~mm}$ was obtained by processing the ultrasonic signals acquired by all sensors (Rx1-Rx8). Reproduced from [24]. 


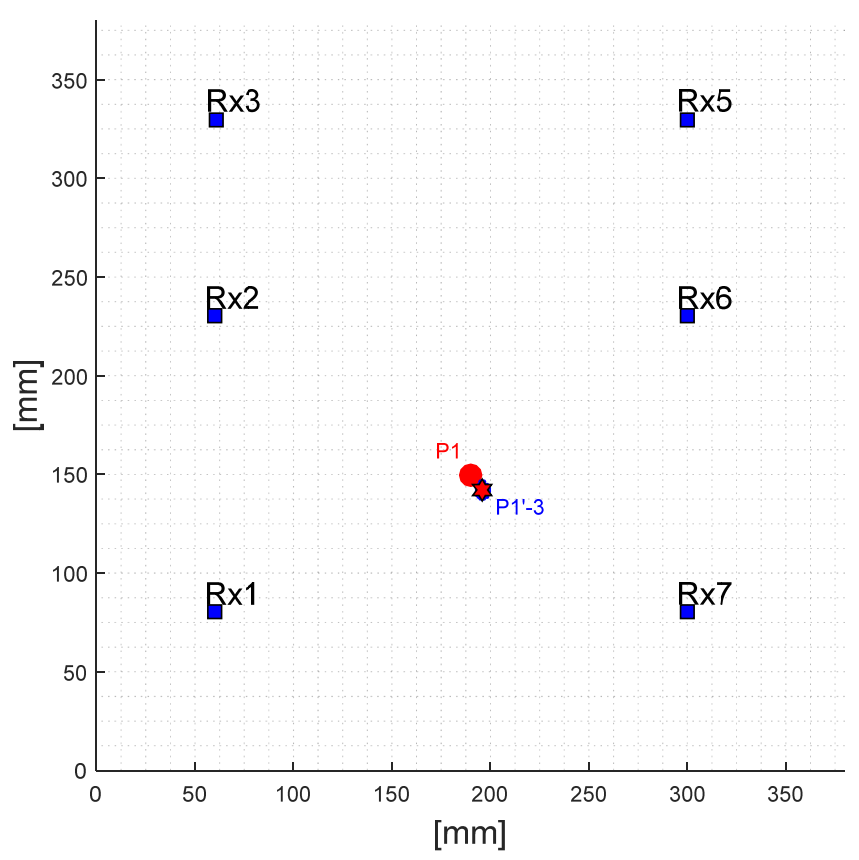

Figure 10. Position of impact $P 1$ (red circle) inside the ROI with coordinates $x_{i}=190 \mathrm{~mm}, y_{i}=150 \mathrm{~mm}$. The detected impact position P1'-3 (red star) with coordinates $\mathrm{x}_{\mathrm{p}}=196 \mathrm{~mm}, \mathrm{y}_{\mathrm{p}}=142 \mathrm{~mm}$ was obtained by processing the ultrasonic signals acquired by six sensors ( $R \times 1, R \times 2, R \times 3, R \times 5, R \times 6$, and $R \times 7)$.

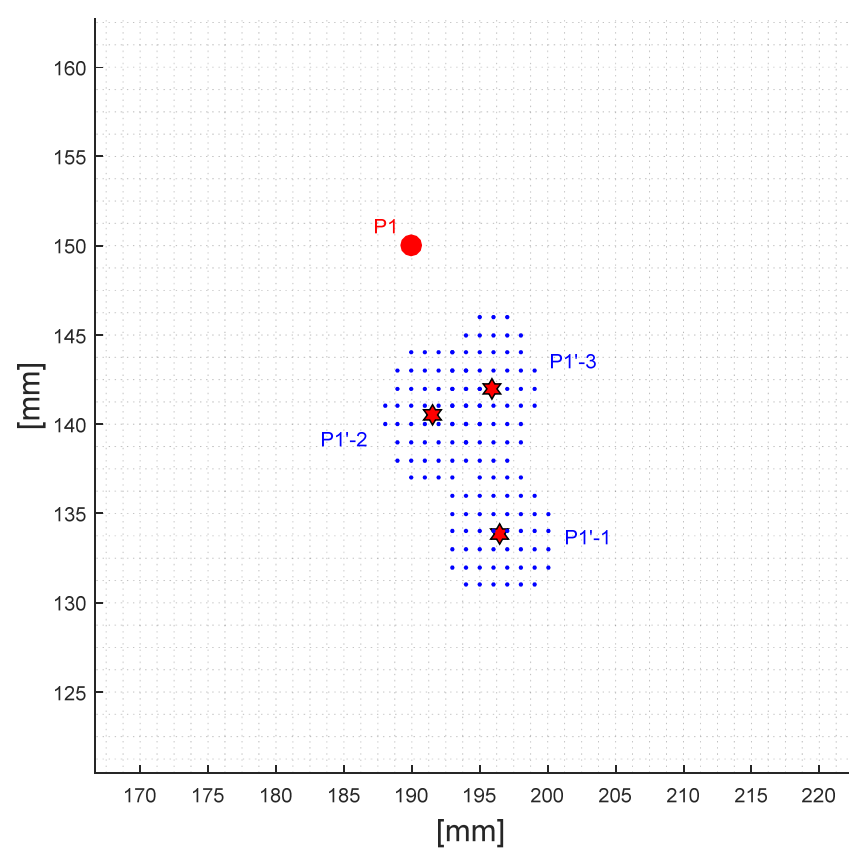

Figure 11. Zoom of results shown in Figures $8-10$. The illustration reports the real impact point P1 (red circle), the detected impact points $\mathrm{P} 1^{\prime}-1, \mathrm{P} 1^{\prime}-2$, and $\mathrm{P} 1^{\prime}-3$ (red stars) together with the points $1 \%$ above the minimum of the error function (blue dots). Reproduced from [24].

Results shown in Figures 8-10 are summarized in Figure 11.

In general, the installation of sensors is based on several constraints imposed by the target structure, while the impact event position is more unpredictable. It is, therefore, worthwhile analyzing impacts outside of the ROI. The full size of the area of the aluminum plate $(500 \mathrm{~mm} \times 500 \mathrm{~mm})$ was divided into a uniform grid of points spaced $1 \mathrm{~mm}$ apart 
with coordinates $\left(\mathrm{x}_{\mathrm{p}}, \mathrm{y}_{\mathrm{p}}\right)$. Figures 12 and 13 show the results obtained by processing the signals acquired from all sensors in the cases of impacts P2 and P3 outside of the ROI.

From Figures 8-11 we can observe that the accuracy of the localization of the impact coordinates is better using eight sensors, compared with using four sensors, but the analysis with six sensors could be a good compromise between sensor density and the error obtained in impact localization. With the eight sensor analysis the localization error was $2 \mathrm{~mm}$ for the $x$-axis and $9 \mathrm{~mm}$ for the $y$-axis, whereas when processing signals acquired by six sensors the error was $6 \mathrm{~mm}$ for the $x$-axis and $8 \mathrm{~mm}$ for the $y$-axis.

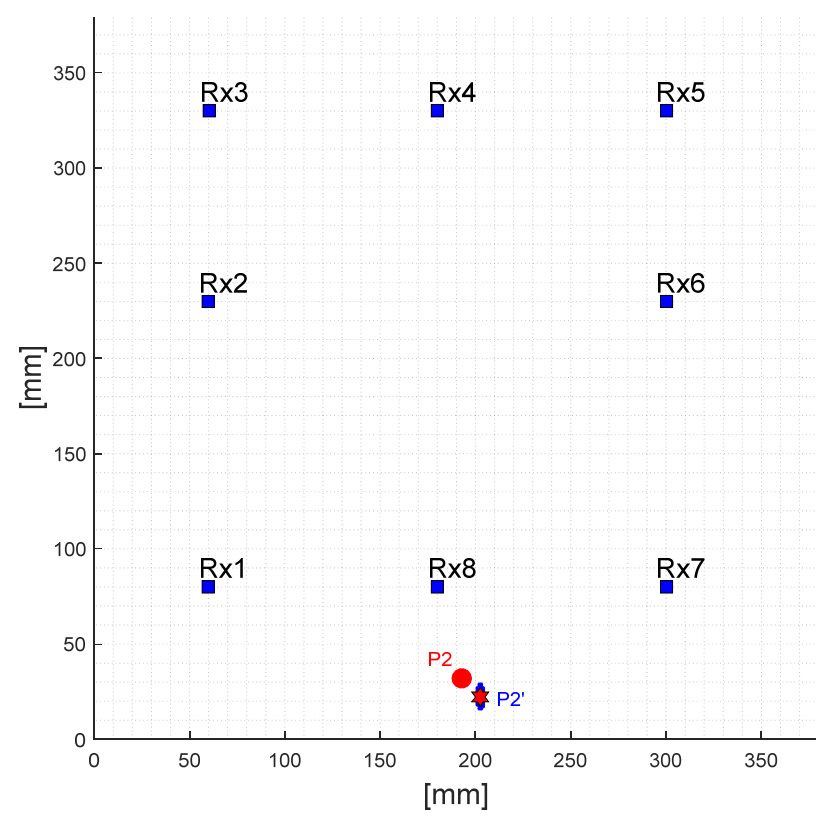

Figure 12. Position of impact P2 (red circle) outside the ROI. The detected impact position P2' (red star) with coordinates $x_{p}=202 \mathrm{~mm}, y_{p}=22 \mathrm{~mm}$ was obtained by processing signals acquired by all sensors. Reproduced from [24].

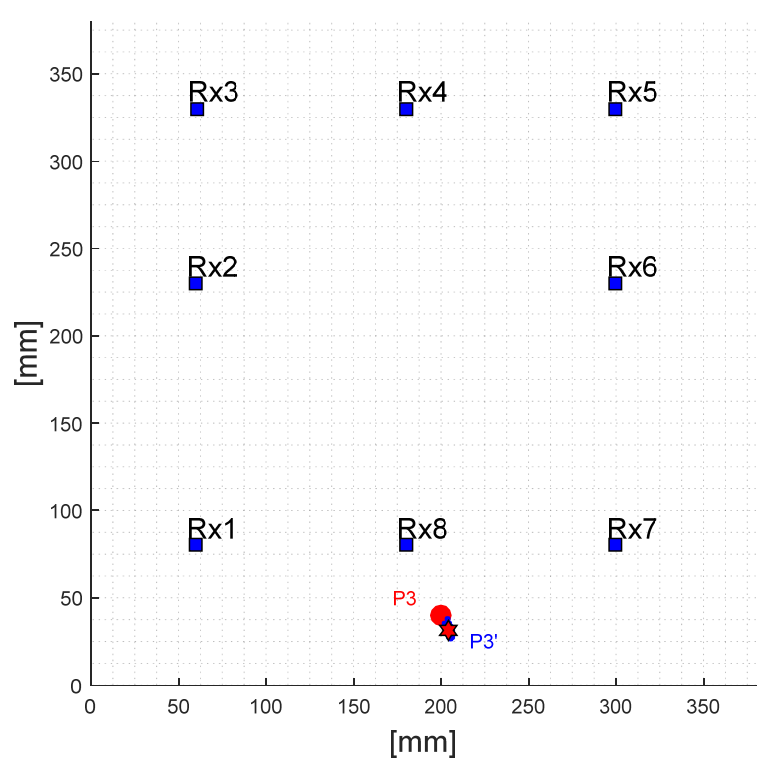

Figure 13. Position of impact P3 (red circle) outside the ROI. The detected impact position P3' (red star) with coordinates $x_{p}=205 \mathrm{~mm}, y_{p}=32 \mathrm{~mm}$ was obtained by processing signals acquired by all eight sensors. 
Figures 12 and 13 demonstrate the ability of the electronic system to reveal, also, impacts outside the ROI with a good accuracy: in both cases, the error in the evaluation of $x$-axis and $y$-axis impact point coordinates was less than $10 \mathrm{~mm}$.

Another observation concerns the placement of sensors. In several real applications, it is not possible to install the sensors on the vertices of regular geometric shapes (squares, circles, hexagons, etc.) and thus positions are decided according to these, and other, constraints. From Figures 8-13 we can observe the position of the eight sensors that are symmetrical along the $x$-axis $(\mathrm{R} \times 1, \mathrm{R} \times 3, \mathrm{R} \times 4, \mathrm{R} \times 5, \mathrm{R} \times 7$, and $\mathrm{R} \times 8)$ but non-symmetric along the $y$-axis ( $R \times 2$ and $R \times 6)$ : these results demonstrate that the calculated impact point is well-identified even in cases of non-symmetric positions of sensors.

The resulting estimates of the three impacts P1, P2, and P3 are summarized in Tables 2-4, respectively. The errors of the impact localization are reported in as a percentage with respect to the dimension of the monitored area, i.e., $500 \mathrm{~mm}$.

Table 2. Estimated impact positions obtained for impact $P 1^{\prime}$ with coordinates $x_{i}=190 \mathrm{~mm}$, $\mathrm{y}_{\mathrm{i}}=150 \mathrm{~mm}$.

\begin{tabular}{cccc}
\hline $\begin{array}{c}\text { Predicted } \\
\text { Impact }\end{array}$ & $\begin{array}{c}\text { Estimated } \\
\text { Position }\end{array}$ & Positioning Error & Error = Positioning Error/500 mm \% \\
\hline \multirow{2}{*}{$1^{\prime}-1$} & $\mathrm{x}=196 \mathrm{~mm}$ & $6 \mathrm{~mm}(x$-axis $)$ & $1.2 \%$ \\
& $\mathrm{y}=134 \mathrm{~mm}$ & $16 \mathrm{~mm}(y$-axis $)$ & $3.2 \%$ \\
$\mathrm{P} 1^{\prime}-2$ & $\mathrm{x}=192 \mathrm{~mm}$ & $2 \mathrm{~mm}(x$-axis $)$ & $0.4 \%$ \\
& $\mathrm{y}=141 \mathrm{~mm}$ & $9 \mathrm{~mm}(y$-axis $)$ & $1.8 \%$ \\
$\mathrm{P} 1^{\prime}-3$ & $\mathrm{x}=196 \mathrm{~mm}$ & $6 \mathrm{~mm}(x$-axis $)$ & $1.2 \%$ \\
& $\mathrm{y}=142 \mathrm{~mm}$ & $8 \mathrm{~mm}(y$-axis $)$ & $1.6 \%$ \\
\hline
\end{tabular}

Table 3. Estimated impact position obtained for impact $P 2^{\prime}$ with coordinates $x_{i}=193 \mathrm{~mm}, \mathrm{y}_{i}=32 \mathrm{~mm}$.

\begin{tabular}{cccc}
\hline $\begin{array}{c}\text { Predicted } \\
\text { Impact }\end{array}$ & $\begin{array}{c}\text { Estimated } \\
\text { Position }\end{array}$ & Positioning Error & Error $=$ Positioning Error $/ \mathbf{5 0 0 ~} \mathbf{~ m m ~ \% ~}$ \\
\hline $\mathrm{P} 2^{\prime}$ & $\mathrm{x}=202 \mathrm{~mm}$ & $9 \mathrm{~mm}(x$-axis $)$ & $1.8 \%$ \\
& $\mathrm{y}=22 \mathrm{~mm}$ & $10 \mathrm{~mm}(y$-axis $)$ & $2.0 \%$ \\
\hline
\end{tabular}

Table 4. Estimated impact position obtained for impact $P 3^{\prime}$ with coordinates $x_{i}=200 \mathrm{~mm}, \mathrm{y}_{\mathrm{i}}=40 \mathrm{~mm}$.

\begin{tabular}{cccc}
\hline Predicted Impact & Estimated Position & Positioning Error & Error $\%$ \\
\hline \multirow{2}{*}{ P3 $^{\prime}$} & $\mathrm{x}=205 \mathrm{~mm}$ & $5 \mathrm{~mm}(x$-axis $)$ & $1.0 \%$ \\
& $y=32 \mathrm{~mm}$ & $8 \mathrm{~mm}(y$-axis $)$ & $1.6 \%$ \\
\hline
\end{tabular}

\section{Discussion and Final Remarks}

This study presents an analysis of errors in impact location with different numbers of channels (from 4 to 8), connected to piezoelectric sensors on an aluminum plate and front-end electronics capable of detecting the early arrival signals of the $\mathrm{S}_{0}$ mode. Impacts were carried out inside and outside of the defined ROI of the plate. To neglect the boundary reflections of the ultrasonic signal due to the free edges of the plate, an adhesive rubber was used with the aim of simulating a semi-infinite space outside the area delimited by the sensors. The DToA were obtained by the threshold method with real-time electronics. The results of the experiments explained well the compromise between sensor density of an SHM system and errors in impact location: the best accuracy was obtained with eight sensors, covering an area of $500 \mathrm{~mm} \times 500 \mathrm{~mm}$ in the aluminum laminate, but a good compromise between sensor density and the error in impact localization was detected when the acquired signal was processed by six sensors-obtaining an error comparable with the assumed wavelength of the $S_{0}$ mode equal to $8 \mathrm{~mm}$. We found that the error was below $3.2 \%$ and slightly better for the $x$-coordinate, which is probably due to a systematic error in 
sensor reference coordinates. The main benefit of the AFE designed for this application is the possibility to select the best signal processing chain to avoid input saturation, with large energy impacts generating 1 to $10 \mathrm{Vpp}$ amplitudes and retaining high SNR, in excess of $72 \mathrm{~dB}$, in the $270-800 \mathrm{kHz}$ bandwidth. We also point out that a certain redundancy in the number of sensors is always useful when considering the possible failure of single channels. Finally, thanks to the programmable configuration of up to 16 channels, this criteria for the selection of optimal sensor density will be investigated also on CFRP plates with the same sensor layout to account for the variability of velocity in composite laminates in different directions.

Author Contributions: Conceptualization, E.M.M. and L.C.; methodology, A.B.; software, E.M.M.; validation, E.M.M. and A.B.; formal analysis, E.M.M.; investigation, A.B. and L.C.; resources, L.C.; data curation, A.B.; writing-original draft preparation, L.C.; writing-review and editing, L.C.; visualization, E.M.M.; supervision, L.C.; project administration, L.C. All authors have read and agreed to the published version of the manuscript.

Funding: This research received no external funding.

Institutional Review Board Statement: Not applicable.

Informed Consent Statement: Not applicable.

Acknowledgments: The authors wish to acknowledge the support of Marco Calzolai, senior technician at the Ultrasound and NDT laboratory of the University of Florence, in conducting this work.

Conflicts of Interest: The authors declare no conflict of interest.

$\begin{array}{ll}\text { Abbreviations } \\ \text { Acronym } & \\ \text { ADC } & \text { Analog-to-digital converter } \\ \text { AFE } & \text { Analog front-end } \\ \text { AI } & \text { Artificial intelligence } \\ \text { CFRP } & \text { Composite fiber reinforce polymer } \\ \text { CWT } & \text { Continuous wavelet transform } \\ \text { DToA } & \text { Differential time of arrival } \\ \text { IDT } & \text { Interdigital transducer } \\ \text { NDT } & \text { Non-destructive testing } \\ \text { PVDF } & \text { Polyvinylidene fluoride } \\ \text { PZT } & \text { Lead zirconate titanate } \\ \text { ROI } & \text { Region of interest } \\ \text { SHM } & \text { Structural health monitoring } \\ \text { SNR } & \text { Signal-to-noise ratio } \\ \text { STFT } & \text { Short-time Fourier transform }\end{array}$

\section{References}

1. Giurgiutiu, V. Structural Health Monitoring: With Piezoelectric Wafer Active Sensors, 2nd ed.; Elsevier: Amsterdam, The Netherlands, 2014; 1024p, ISBN 9780124186910.

2. Rose, J.L. Ultrasonic Guided Waves in Solid Media; Cambridge University Press: Cambridge, UK, 2014.

3. Ostachowicz, W.; Kudela, P.; Krawczuk, M.; Zak, A. Guided Waves in Structures for SHM: The Time-Domain Spectral Element Method; John Wiley \& Sons: New York, NY, USA, February 2012; 350p, ISBN 978-0-470-97983-9.

4. Purekar, A.S.; Pines, D.J. Damage detection in thin composite laminates using piezoelectric phased sensor arrays and guided lamb wave interrogation. J. Intell. Mater. Syst. Struct. 2010, 21, 995-1010. [CrossRef]

5. Qiu, L.; Deng, X.; Yuan, S.; Huang, Y.; Ren, Y. Impact monitoring for aircraft smart composite skins based on a lightweight sensor network and characteristic digital sequences. Sensors 2018, 18, 2218. [CrossRef] [PubMed]

6. Tobias, A. Acoustic-emission source location in two dimensions by an array of three sensors. Non-Destr. Test. 1976, 9, 9-12. [CrossRef]

7. Ciampa, F.; Meo, M. A new algorithm for acoustic emission localization and flexural group velocity determination in anisotropic structures. Compos. Part A Appl. Sci. Manuf. 2010, 41, 1777-1786. [CrossRef] 
8. Mohd, S.; Holford, K.M.; Pullin, R. Continuous wavelet transform analysis and modal location analysis acoustic emission source location for nuclear piping crack growth monitoring. In Proceedings of the AIP Conference Proceedings, Punjab, India, 17-21 December 2014; pp. 61-68.

9. Yin, S.; Cui, Z.; Kundu, T. Acoustic source localization in anisotropic plates with "Z" shaped sensor clusters. Ultrasonics 2018, 84, 34-37. [CrossRef] [PubMed]

10. Worden, K.; Staszewski, W.J. Impact location and quantification on a composite panel using neural networks and a genetic algorithm. Strain 2000, 36, 61-68. [CrossRef]

11. Carrino, S.; Nicassio, F.; Scarselli, G. An innovative method based on nonlinear Lamb waves for locating disbonds in Single-Lap joints. In Proceedings of the 2019 IEEE 5th International Workshop on Metrology for AeroSpace (MetroAeroSpace), Torino, Italy, 19-21 June 2019; pp. 187-191.

12. Carrino, S.; Nicassio, F.; Scarselli, G. Non-linear Lamb Waves for Locating Defects in Single-Lap Joints. Front. Built Environ. J. 2020, 6. [CrossRef]

13. Kundu, T. Acoustic source localization. Ultrasonics 2014, 54, 25-38. [CrossRef] [PubMed]

14. Sen, N.; Kundu, T. A new wave front shape-based approach for acoustic source localization in an anisotropic plate without knowing its material properties. Ultrasonics 2018, 87, 20-32. [CrossRef] [PubMed]

15. Bulletti, A.; Giannelli, P.; Calzolai, M.; Capineri, L. An Integrated Acousto/Ultrasonic Structural Health Monitoring System for Composite Pressure Vessels. IEEE Trans. Ultrason. Ferroelectr. Freq. Control 2016, 63, 864-873. [CrossRef] [PubMed]

16. Merlo, E.M.; Bulletti, A.; Giannelli, P.; Calzolai, M.; Capineri, L. An Integrated Acousto/Ultrasonic Structural Health Monitoring System for Composite Pressure Vessels, A Novel Differential Time-of-Arrival Estimation Technique for Impact Localization on Carbon Fiber Laminate Sheets. Sensors 2017, 17, 2270. [CrossRef] [PubMed]

17. Merlo, E.M.; Bulletti, A.; Giannelli, P.; Calzolai, M.; Capineri, L. Analysis of Errors in the Estimation of Impact Positions in Plate-Like Structure through the Triangulation Formula by Piezoelectric Sensors Monitoring. Sensors 2018, 18, 3426. [CrossRef] [PubMed]

18. Lehmann, M.; Büter, A.; Frankenstein, B.; Schubert, F.; Brunner, B. Monitoring System for Delamination Detection-Qualification of Structural Health Monitoring (SHM) Systems. In Proceedings of the Conference on Damage in Composite Materials (CDCM), Stuttgart, Germany, 18-19 September 2006.

19. Ono, K. Review on Structural Health Evaluation with Acoustic Emission. Appl. Sci. 2018, 8, 958. [CrossRef]

20. Xinlin, Q.; Wenzhuo, L.; Yishou, W.; Hu, S. Piezoelectric Transducer-Based Structural Health Monitoring for Aircraft Applications. Sensors 2019, 19, 545. [CrossRef]

21. Capineri, L.; Bulletti, A.; Calzolai, M.; Giannelli, P.; Francesconi, D. Arrays of conformable ultrasonic Lamb wave transducers for structural health monitoring with real-time electronics. Procedia Eng. 2014, 87, 1266-1269. [CrossRef]

22. Ebrahimkhanlou, A.; Dubuc, B.; Salamone, S. A generalizable deep learning framework for localizing and characterizing acoustic emission sources in riveted metallic panels. Mech. Syst. Signal Process. 2019, 130, 248-272. [CrossRef]

23. Ross, R. Structural health monitoring and impact detection using neural networks for damage characterization. In Proceedings of the 47th AIAA/ASME/ASCE/AHS/ASC Structures, Structural Dynamics, and Materials Conference 14th AIAA/ASME/AHS Adaptive Structures Conference 7th, Newport, RI, USA, 1-4 May 2006; p. 2117.

24. Bulletti, A.; Merlo, E.M.; Capineri, L. Analysis of the accuracy in impact localization using piezoelectric sensors for Structural Health Monitoring with multichannel real-time electronics. In Proceedings of the 2020 IEEE 7th International Workshop on Metrology for AeroSpace (MetroAeroSpace), Pisa, Italy, 22-24 June 2020. 